\title{
Design Of Remote Elevator Monitor Systembased On Coldfire Microcontroller
}

\author{
Zhengping Li \\ School of Electronics and Information Engineering \\ Anhui University \\ Hefei, China \\ e-mail: bluefeynman@gmail.com
}

\author{
Weiwei Wang \\ School of Electronics and Information Engineering \\ Anhui University \\ Hefei, China \\ e-mail:07ahuwww@163.com
}

\begin{abstract}
The paper introduces the design of elevator monitor system, and the system can monitor the running status of the elevator and ensure the safety of the elevator. The system is based on Freescale corporation MCF51AC128 Coldfire microcontroller. We adopted Free Real Time Operating System (FreeRTOS) as software platform. We adopted General Packet Radio Service (GPRS) and Controller Area Network (CAN) bus technology to implement remote elevator monitor, and users can choose according to their requirement. In addition, the system used capacitance isolation technique to design CAN bus interface and software control flow error check technique to assure reliability. The application shows that the system runs stably. The system is very cost effective and easy to use.
\end{abstract}

Keywords-elevator monitor; Coldfire; capacitance isolation; Free Real Time Operating System (FreeRTOS); control flow error checking

\section{INTRODUCTION}

With the social development, the elevator safety problems attract more and more attention. Although elevator companies have their own monitoring systems, these systems are only developed for their respective brands and are not compatible. In practical applications, such systems are inconvenient to use and are in the idle state substantially.

This paper introduces a remote elevator monitoring system design which is based on Freescale Coldfire micro controller and Free Real Time Operating System (FreeRTOS) real-time operating system. Compared to conventional solution based on Programmable Logic Controller (PLC), this design has lowcost and cost-effective advantages. The system uses optical isolation technology to collect input signals of the sensor. By analysing signals of the sensor, grasp the operation state of the elevator accurately and record in real time. When the elevator runs abnormally, the system realizes remote alarm and monitoring through General Packet Radio Service (GPRS) and combines Controller Area Network (CAN) bus to achieve a wired remote monitoring. GPRS is a continuation of the GSM (Global System for Mobile Communications). GPRS transmits with the packet type. The complete system consists of Personal
Computer (PC) management software and the elevator monitoring equipment.

This paper mainly expounds the design of the elevator monitoring equipment.

\section{SYSTEM FRAMEWORK}

Elevator monitoring device is based on Freescale industryleading Coldfire microcontrollers MCF51AC128 [1, 2]. The device integrates GPRS wireless communications, Moving Picture Experts Group Audio Layer-3 (MP3), Secure Digital Memory Card (SD card) storage, CAN, Recommend Standard 232 (RS232), sensor signal detection and so on which are placed inside the elevator.

Sensor module includes two photoelectric switches which are fixed on the elevator to detect position and motion state of an elevator. One is body pyroelectric infrared sensor which is used to detect whether someone is inside the elevator, the other sensor detects whether the door of elevator is closed. The output of the two limit switches and the sensors is switch signal. The two limit switches are used to test whether elevators reach the top and the bottom, and a number of the sensors test safety circuit.

Two photoelectric switches are fixed on the elevators, and one above and one below. Each floor is set up a baffle. When the elevators pass, the upper and lower photoelectric switches are blocked by baffles, resulting in two switch signals. By detecting the signals and the sequence, Software judges the floor of the elevator as well as the direction and speed of the operation.

System software consists of FreeRTOS operating system, driver, File Allocation Table File System (FATFS) file system and the auxiliary libraries [3]. System software contains each concrete hardware module driver and FATFS file system which is used to manage an SD card. In addition it also contains a concise standard C library to provide string operations, memory allocation and other necessary functions. On the basis, develop the application software to accomplish specific functions. 


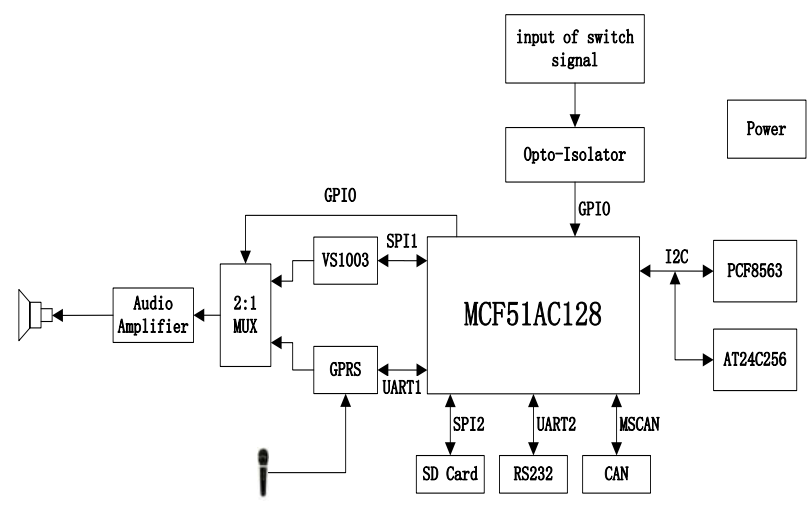

Figure 1. The system hardware diagram

\section{HARDWARE DESIGN}

This system adopts 50 MHZ MCF51AC128 micro controller. Respectively extend an SD card and VS1003 MP3 decoding chip through two integrated Serial Peripheral Interface (SPI) controller to realize large-capacity storage of the operating data and MP3 files and MP3 decoding playing. Connect Inter-Integrated Circuit (I2C) with PCF8563 real time clock to record the system time. In addition, the system extends a piece of AT24C256 Electrically Erasable Programmable Read - Only Memory (EEPROM) of I2C interface to hold the system critical configuration data, such as the Internet Protocol (IP) address of GPRS module and the gateway address.

There are two Universal Asynchronous Receiver Transmitter (UART) modules of MCF51AC128. One is for communication with the GPRS module, and the other realizes RS232 interface through which the user can connect the PC to configure the system related parameters, such as changing the alarm telephone number, setting the device address etc. Use General Purpose Input/ Output (GPIO) to realize sensor switch signal detection.

The system hardware block diagram is shown in Fig.1.

Power affects the reliability and stability of the overall system directly, due to large range of external input supply voltage Vext $(10 \mathrm{~V} \sim 24 \mathrm{~V})$ and low quality of power. But the system hardware needs various supply voltage within the range of $2.5 \mathrm{~V} \sim 5 \mathrm{~V}$. We use LM2576 DC-DC switch power supply chip to generate $5 \mathrm{~V}$ (VCC5) for MP3 module audio amplifier circuits. Considering MCF51AC128 and GPRS module level matching, the system uses LM2576 to generate 5V voltage. The $5 \mathrm{~V}$ voltage is generated a stable $3.1 \mathrm{~V}$ by LM1117 Low Dropout Regulator (LDO) to supply the main chips of system. GPRS module requires $3.3 \mathrm{~V} \sim 4.4 \mathrm{~V}$ power supply. When the peak currents of sending and receiving data are up to $2 \mathrm{~A}$, the system uses the Sipex Corporation SPX29302 LDO to generate $4.0 \mathrm{~V}$ directly from the Vext for GPRS modules. In addition, $2.5 \mathrm{~V}$ which supply VS1003 decoder chip is generated from VCC5 directly.

In order to improve stability and anti-jamming capability of the system, we use optocoupler isolation technology to collect signals of external switch quantity sensor, including detecting leveling, safety circuit, door locks and other states. Thus, track and record the current operating status of the elevator accurately.

System uses Siemens MC52i GPRS module and Texas Instruments (TI) ISO1050 isolated CAN transceiver to communicate with residential property or other managements.
The ISO1050 is a galvanically isolated CAN transceiver that

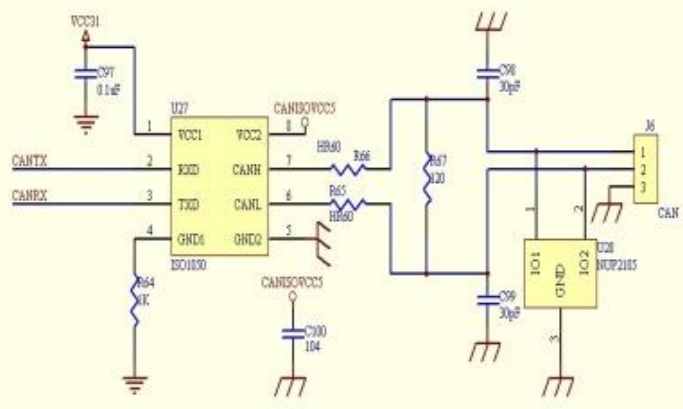

Figure 2. Isolation CAN interface circuit

meets or exceeds the specifications of the ISO11898 standard.The device has the logic input and output buffers separated by a silicon oxide( $\mathrm{SiO} 2)$ insulation barrier that provides galvanic isolation of up to 4000 VPEAK. As a CAN transceiver, the device provides differential transmit capability to the bus and differential receive capability to a CAN controller at signaling rates up to 1 megabit per second(Mbps) [4].

Fig. 2 shows the CAN interface circuit diagram. ISO1050 has $4000 \mathrm{~V}$ isolation voltage and conforms to ISO11898 standard, and the communication speed is up to $1 \mathrm{Mbps}$, which greatly simplifies the traditional CAN-bus isolation design scheme which is based on optocoupler [4]. System adopts Positive Temperature Coefficient (PTC) resistor HR60 for overcurrent protection and adopts NUP2105 bidirectional Transient Voltage Suppressors (TVS) of ON Semiconductor for overvoltage protection. In addition, for providing the isolation power supply, the system adopts ZY0505BS-1w micro-power isolated power supply module of Zhou Ligong microcontroller technology co., LTD to generate isolated $5 \mathrm{~V}$ power for ISO1050.The isolated $5 \mathrm{~V}$ power is from $5 \mathrm{~V}$ power supply which is the output of LM2576.

Mp3 decoding uses VS1003 to achieve, and its output is played by the audio amplifier drive speakers. When the elevator fault occurs, the system will automatically play an $\mathrm{mp} 3$ audio files which is pre-stored in the SD card to calm trapped people. Audio power amplifier is shared by VS1003 and GPRS. VS1003 and GPRS switch through a relay. When mp3 audio files play, MCF51AC128 gates VS1003 output to audio power amplifier through GPIO. On the contrary, when the voice calls, gate GPRS voice output to audio power amplifier. System sets a microphone in the elevator for voice calls.

\section{SOFTWARE DESIGN}

The system software is mainly divided into two layers: system software layer and application software layer. System software layer mainly includes FreeRTOS, FATFS file system, streamlining $\mathrm{C}$ standard library, and each module driver of the hardware. Application software layer is based on the services which are provided by the system software layer to complete specific functions of elevator monitoring system. 


\section{A. System Software Design}

FreeRTOS is a small embedded system which has mini operating system kernel. As a lightweight operating system, features include: task management, time management, semaphores, message queues, memory management, logging, etc. It can basically meet the needs of smaller systems.

FreeRTOS is a lightweight real-time kernel. It supports priority-based scheduling and round-robin scheduling which is based on the time slice. It supports preemptive kernel and collaborative kernel. Relative to domestic popular $\mu \mathrm{C} / \mathrm{OS}-\mathrm{II}$, it is completely free and open-source. The system real-time performance is equivalent with $\mu \mathrm{C} / \mathrm{OS}-\mathrm{II}$, but resources consume fewer.

This system transplants FreeRTOS on MCF51AC128 and uses on-chip timer of MCF51AC128 to generate the operating system clock tick. The system clock tick is $20 \mathrm{~ms}$. In addition, we implement a streamlined $\mathrm{C}$ library. Only provide the $\mathrm{C}$ standard library functions which the system needs. Therefore, greatly reduce the size of the final object code $[5,6]$.

\section{B. Application Software Design}

Application software relies on the services which are provided by the system software to realize the function of customer demand. After the system reset and system initialization of the main function, create six FreeRTOS tasks and start FreeRTOS scheduler. Thereafter, FreeRTOS which is based on priority schedules tasks to complete the corresponding functions.

Sensor_task queries and analyzes input signals once every $500 \mathrm{~ms}$. If it is found that the elevator operation is abnormal, wake warn_task to alarm through message queue. If there are people exist in the elevator when the elevator detects abnormally, the system wakes mp3_task to play appeasing music. If it is detected that emergency call button in the elevator is pressed, sensor_task determines whether the current state of the elevator is normal. If it is not normal, automatically call management center telephone. In addition, the task also regularly stores running status of the elevator to the SD card.

Warn_task is responsible for sending out alarm signal through GPRS and CAN when an exception of the elevator occurs. During normal operation of the elevator, the task is in pending state [7,8]. The functions of gprs_task and can_task are similar. The former is responsible for monitoring input data of GPRS on the line, and the latter is responsible for monitoring the data on the CAN bus. When data are received, identify the data. If it is a valid command, then process it. Commands include that the host computer queries current state of the elevator, phone calls, setting up the system parameters and so on. When receiving a telephone call of management center, gprs_task hangs mp3_task. Switch audio output of GPRS to the audio amplifier circuit and turn on the phone to talk with people in the elevator.

Mp3_task is responsible for playing the audio files in SD card. Sensor_task will wake mp3_task to play music, when people are trapped in an elevator. On the one hand it can help trapped people save themselves. On the other hand it can help appease the trapped people.

RS232_task achieves to configure the system via RS232 for users. This task is usually in a suspended state. Once the data are received, UART interrupt service routines wake up the task to deal with configuration command of the PC.

\section{Program Control Flow Error Checking Technology}

In order to improve the stability and reliability of the entire system, we use a pure software method called Relationship Signatures for Control Flow Checking (RSCFC) to test program flow errors. The basic idea is to divide tasks into a number of basic blocks. Each basic block is assigned a number. In the beginning and the end position of each basic block, insert error detection code which is used to judge whether the execution flow of the program occurs errors due to interference. The basic block refers to a code block without switch-case, jump codes. That is, in the basic block except the last one instruction, it does not include any instruction such as jump, switch-case or call instruction which changes control flow. Program control flow can be described using the jump relation between each basic block. For example, basic block could jump into the block j1, j2...jm. I is called the predecessor block of these blocks. Andj1, etc are the subsequent blocks of $i[9,10]$.

The jump relation can be determined in advance. So, record the block number at the end of the each basic block. When entering into the next basic block, detect whether the current basic block has a valid subsequent block. If not, the control flow error occurs. For example, record number $i$ at the end of the basic block $\mathrm{i}$. When entering into the next basic block $\mathrm{j}$, determine whether $\mathrm{j}$ is one of valid subsequent blocks $\mathrm{j} 1$ 、 $\mathrm{j} 2 、 \ldots$ jm of i. If not, error must happen.

Once the control flow error is detected, the system immediately disables interrupts and sends error alarm via GPRS and the CAN. At the same time, record the error log in the SD card. Then restart the system.

\section{CONCLUSION}

The system of elevator monitoring enhances the safety of the elevator. When a failure occurs, it can alarm in time to protect the personal safety of users.

This paper describes a system design which is based on micro controller MCF51AC128 and FreeRTOS. The system uses GPRS and CAN bus to realize remote monitoring. The system design fully considers requirements of the antiinterference and reliability. The system has been tried at the scene half a year. The system running is stable, and evaluations of users are good.

\section{ACKNOWLEDGMENT}

This work is supported by the China National Science and Technology major Project (2011ZX01034-001-002-003).

\section{REFERENCES}

[1] Freescale Corp, MCF51AC128 reference manual, http://cache.Freescale.com/files/32bit/doc/data_sheet/MCF51AC256.pdf.

[2] Chen Yimei and YouYiMing, "Freescale flexis series microcontroller introduction and development," Electronic Industry Press, 2009.

[3] chaN, FatFS generic file system module, http://elmchan.org/fsw/ff/00index_e.html.

[4] Texas Instruments Corporation, ISO1050 datasheet, http://www.ti.com/lit/gpn/iso1050.

[5] FreeRTOS API Manual, http://www.freertos.org.

[6] Anonymous, "Real time engineers releases FreeRTOS v6," Wireless News, 2010. 
[7] Nikolas V. Stylianides, George V. Stephanopoulos, George S. Tselikis, and Michael Dopher, "Signaling performance trials and evaluation results on a GPRS platform," Information Systems Frontiers, vol. 72, 2005.

[8] Yieh-Ran Haung and Jan-Ming Ho, "Overload control for short message transfer in GPRS/UMTS networks," Information Sciences, 2004, pp. 1702 .

[9] Bruce P. Lester, "Detection of control flow errors in parallel programs at compile time," International Journal of Distributed and Parallel Systems, December 2010.

[10] LI Ai-guo and HONG Bing-rong, "A software method for on-line control flow fault detection," Journal of Astronautics, pp.1424-1430, June 2006. 